\title{
Shocks in the Quiet Solar Photosphere: A Rather Common Occurrence
}

\author{
H. Socas-Navarro and R. Manso Sainz \\ High Altitude Observatory, NCAR, 3450 Mitchell Lane, Boulder, CO 80307-3000, USA \\ navarro@ucar . edu
}

\begin{abstract}
We present observations of the quiet solar photosphere in the Fe I lines at $6302 \AA$ where at least four different spatial locations exhibit upwards-directed supersonic flows. These upflows can only be detected in the circular polarization profiles as a double-peaked structure in the blue lobe of both Fe I lines. We have detected cases of either magnetic polarity in the data. The polarization signals associated with the upflows are very weak, which is probably why they had not been seen before in this type of observations. We propose that the observed flows are the signature of aborted convective collapse, similar to the case reported by Bellot Rubio et al. (2001). Our data indicates that this phenomenon occurs frequently in the quiet Sun, which means that many magnetic elements (although the fraction is still unknown) are destroyed even before they are formed completely. The spectral signatures of supersonic upflows reported here are probably present in most spectro-polarimetric observations of sufficient signal-to-noise and spatial resolution.
\end{abstract}

Subject headings: line: profiles - Sun: atmospheric motions - Sun: magnetic fields - Sun: photosphere stars: atmospheres

\section{Introduction}

The term quiet Sun is commonly used to refer to the solar atmosphere away from sunspots, faculae and other large-scale concentrations of magnetic flux. The adjective "quiet", however, seems now less of a synonym of "unintersting" than it did only a few years ago. This is due to both recent numerical simulations (e.g., Emonet \& Cattaneo 2001; Carlsson et al. 2004) and new spectro-polarimetric observations of high sensitivity and resolution (e.g., Sánchez Almeida \& Lites 2000; Socas-Navarro \& Sánchez Almeida 2002; Khomenko et al. 2003; Domínguez Cerdeña et al. 2003). From the point of view of the magnetic energy balance it is worth mentioning that, according to these works, there is probably more (unsigned) magnetic flux in the quiet Sun than in all the active regions during solar maximum. The

\footnotetext{
${ }^{1}$ The National Center for Atmospheric Research (NCAR) is sponsored by the National Science Foundation.
}

small-scale structure of the magnetic elements is also a hot topic of debate (e.g., Socas-Navarro \& Sánchez Almeida 2003).

The present work adds to this more exciting view of the quiet photosphere by reporting on the detection of supersonic upflows, which seem to be a relatively frequent event. We argue that these flows are likely the last stages of the process observed by Bellot Rubio et al. (2001) (hereafter BRCKR) in the infrared. According to their scenario, the upflows are the signature of an aborted convective collapse and subsequent disintegration of the magnetic structure. Our observations support this picture to some extent and remove any caveats that might have existed on their observation (e.g., structures moving in or out of the slit).

\section{Observations}

The observations used in this paper were taken with the Advanced Stokes Polarimeter (ASP, El- 
more et al. 1992) and first analyzed by Lites (1996). The strong upflows that we report here have gone unnoticed until now because their associated polarization signals are very weak. The amplitudes of Stokes $V$ are at most of the order of $5 \times 10^{-3}$, and even weaker in some of the interesting points (at the $10^{-3}$ level). This value is close to the noise level of the observations $\left(5 \times 10^{-4}\right)$, but fortunately it is high enough that it allows for a few evident detections.

Figure 1 shows a map of the continuum intensity in the observed region (left) and the degree of circular polarization (right). The four spatial locations of regions where we have found clear detections are marked with $\mathrm{A}, \mathrm{B}, \mathrm{C}$ and $\mathrm{D}$, respectively. We have found some other pixels where the profiles seem to exhibit a similar structure, but the signal-to-noise ratio is not good enough to be sure. Three of the detections have the same polarity (regions $\mathrm{A}, \mathrm{B}$ and $\mathrm{C}$ ), whereas the forth one (D) has the opposite.

The Stokes $I$ and $V$ profiles corresponding to the best two detections are plotted in Fig 2. The intensity profiles do not show any distinctive features. However, the Stokes $V$ spectra show a double-peaked structure in the blue side of the line. Observational studies of Stokes $V$ asymmetries in the quiet Sun show that the red lobe is typically broader and shallower than the blue one (e.g., Sánchez Almeida \& Lites 2000), which explains why the double peak is not visible in the red lobe of the lines. The zero-crossing wavelength of the overall profile is practically at the same position as the intensity minimum. The difference between the two blue peaks in both lines corresponds approximately to $7.5 \mathrm{~km} \mathrm{~s}^{-1}$.

These two profiles were the first that we identified in the map by visual inspection. We developed an automated search algorithm to try to find other similar occurrences in the map. The algorithm looks for extended blue wings in both Fe I lines. The candidates detected in this manner have been identified in Fig 1.

The upflow in region A spans an area large enough that we can improve the signal-to-noise ratio considerably by binning the profiles spatially. Using a $4 \times 4$ box average we were able to detect some linear polarization signal in Stokes $U$ (Stokes $Q$ is still within the noise after binning). Figure 3 shows the binned Stokes $V$ and $U$. It is interesting to note that the upflowing component does not have any linear polarization associated to it in either Fe I line.

We also looked for profiles similar to these in other high-resolution quiet Sun observations. For this purpose we analyzed the three maps observed by Lites \& Socas-Navarro (2004) with the Diffraction-Limited Stokes Polarimeter (Sankarasubramanian et al. 2003). Although those maps have very good spatial resolution $(\simeq 0.6 ")$, we could not find any obvious occurrence. We believe the reason is the lower signal-to-noise ratio in the DLSP data (about a factor 3 worse).

\section{Interpretation}

The most natural explanation for the doublepeaked structure in Stokes $V$ is the presence of two atmospheric components with very different velocities along the line of sight (i.e., in the vertical direction, since these observations were taken at disk center). Inspection of the Stokes $V$ spectra along the slit shows that the red component of the double peak is nearly at rest with respect to the other magnetic signals. We shall refer to this as the "normal" component. The blue component is shifted in wavelengths by at least $157 \mathrm{~m} \AA$ (the separation between the peaks), or approximately $7.5 \mathrm{~km} \mathrm{~s}^{-1}$. This will be referred to as the "upflowing" component.

For a more detailed study, we carried out inversions of the average profiles in region $\mathrm{A}$. We used a modified version of the LTE code LILIA (Socas-Navarro 2001; see also Ruiz Cobo \& del Toro Iniesta 1992) that considers two magnetic atmospheres embedded in an unmagnetized background. In addition to the usual magnetic filling factor $\alpha$, we now have the individual filling factors of the two magnetic components. The nonmagnetic component is assumed to produce an average unpolarized quiet Sun profile. The two magnetic components are allowed to have different temperature, density, line of sight velocity, magnetic field vector (strength and orientation) and microturbulence. These parameters are recovered independently by the inversion code as a function of height.

The models obtained from the inversion, as well as the fits to Stokes $I$ and $V$, are presented in Fig 4. While the normal component exhibits a 
rather smooth behavior with more or less constant properties, the upflowing component shows some interesting properties. The temperature stops decreasing with height and even increases considerably above $z=200 \mathrm{~km}$, approximately. The magnetic field, which has a more or less constant value of $500 \mathrm{G}$ in the normal component, has a sharp discontinuity going from zero to $1 \mathrm{kG}$ between $z=150$ and $z=200 \mathrm{~km}$. Finally, the line of sight velocity goes from a moderate upflow the bottom of the photosphere to reach values of $\simeq 10 \mathrm{~km} \mathrm{~s}^{-1}$ at $z=200 \mathrm{~km}$. Remarkably, the relative velocity between the two atmospheres turns from subsonic to supersonic precisely at $z=200 \mathrm{~km}$.

All the properties mentioned above indicate the presence of a shock front propagating upwards in the photosphere. The coincidence between the three curves in the middle right panel of Fig 4 (within a few $\mathrm{km}$ ) is remarkable. This means that the point where the upflowing atmosphere is heated by the shock and its temperature starts to increase is also the point where the upflow reaches the sound speed in the medium. Since the three curves are derived independently of each other, this is a good consistency test for the reliability of the inversion. The strong gradients in the magnetic field and velicity (present only in the upflowing component) are also consistent with the shock scenario.

We find that the observations described here bear a striking resemblance to the infrared ones of BRCKR near $1.6 \mu \mathrm{m}$. Their analysis of fixedslit time-series of a quiet Sun region lead them to conclude that they had observed an instance of aborted convective collapse. After an initial stage of field concentration with moderate downflows, the magnetic structure quickly disintegrated presumably destroyed by an upwards-propagating shockwave.

We propose that the double-peaked blue lobes of the profiles that we have detected are the visible counterparts of the last stages of evolution in the BRCKR observations. In principle there is no reason why the process they observed would be more easily detectable in the infrared than in the visible. One might argue that the Doppler shift increases linearly with wavelength, but on the other hand so does the thermal width of spectral lines. Therefore, the relative velocity needed to produce a double-peaked Stokes $V$ lobe would be approx- imately the same in the visible and the infrared, especially considering that the spectral lines observed are from the same ion.

The area fraction occupied by pixels harboring shocks in our map is $f=7 \times 10^{-4}$. Let us assume, for the sake of argument, that the convective collapse observed by BRCKR is "typical" and take the duration of the entire process to be 15 minutes and the upflowing phase 3 minutes (as reported by them). With these parameters we can estimate that the probability of these authors to observe an aborted convective collapse in their 60 minute time-series of a $30 \times 0.5$ arc-seconds area is approximately 0.45 . This figure supports the idea that we have observed the same phenomenon.

The absence of linear polarization in the upflowing component (Fig 3), while the quiet component exhibits some signal, is surprising. In principle there are two possible explanations for this. The most obvious would be that the upflow disrupts the initial organization of the field in the horizontal direction. A second possibility is that the field may be weaker in the upflowing component, reducing the linear polarization below the detection threshold. Notice that Stokes $V$ can still have a similar amplitude in both components because it would be compensated by a hypothetical temperature (and thus also source function) increase in the upflow. This explanation is plausible because, while Stokes $V$ has a first-order dependence on the field strength, the dependence of Stokes $Q$ and $U$ is of second order. The response to temperature (through the source function) is the same in all Stokes parameters. Since temperature and field strength affect linear and circular polarization differently, it is possible to find a suitable combination that preserves one and destroys the other.

\section{Conclusions}

The observations presented in this paper reveal supersonic upflows in the quiet solar photosphere and indicate that this is a rather common occurrence. The upflows are probably the signature of an exploding magnetic element after an aborted convective collapse proposed by BRCKR. It is important to point out that we do not observe the flux destruction directly. However, the assumption that we are observing the same process seems appropriate based on the following facts: 
- The only observations reported in the literature with upflows inside photospheric magnetic elements is the aborted convective collapse of BRCKR.

- The magnitude of the upflows is the same, very close to the sound speed of the photosphere at the heights where the lines form. This is what one would expect from a shockwave propagating inside the magnetic element.

- If one assumes that we are observing the same process and that their collapse time scales are typical, it is possible to estimate the probability that BRCKR observed an aborted convective collapse in their timeseries. We find that this probability is of the order of 1, which would be an unlikely coincidence if our assumption were incorrect.

From a 2-component inversion of the doublepeaked profiles we obtain a model atmosphere that is consistent with a shockwave propagating in one of the components. The inversion even retrieves a zero field below the front shock, which would also be in agreement with the destruction of the magnetic element reported by BRCKR.

Interestingly, we only observe instances in which the two atmospheric components have similar Stokes $V$ amplitudes. A possible explanation to this could be the following. The upflowing component cannot have a larger amplitude because, if it is indeed an exploding magnetic element, the field must be weaker in this component. If, on the other hand, it had a significantly lower amplitude then its Stokes $V$ profile would be buried in the blue lobe of the quiet component and we would not be able to see it. Therefore, it is likely that we are only observing a small fraction of all exploding elements.

Because of the selection effect mentioned above and the fact that we are close to the sensitivity limit of the data, the frequency of occurrence reported here should be taken as a (perhaps severley underestimated) lower limit.

Finally, we find that the spatial extent of the upflows are quite puzzling, especially region A. The upflows span here a significantly large area of more than 2 by 2 arc-seconds. Considering that the magnetic filling factor is very small in this area $(\sim 10 \%)$, this is most certainly not a single magnetic structure disintegrating. However, if this area is composed of a collection of small magnetic elements (as is generally thought that network elements are), then what physical mechanism would syncrhonize the exploding elements as far as 2 arcseconds apart?

It is important to continue investigating these upflows in more detail, given the potential implications of this work on the generation and destruction of small-scale magnetic elements in the quiet Sun. As mentioned earlier, these fields may be carrying most of the solar magnetic flux. It is fundamental to determine whether convective collapse is an efficient mechanism for concentrating field and these aborted instances constitute only a small fraction of the cases, or if on the contrary most (or perhaps even all) of the collapsing elements are ultimately doomed to disintegrate sending shockwaves into the photosphere. The amount of energy deposited in the photosphere, and also in the upper atmosphere, by the shocks might be important if a large number of these events are taking place continuously in the quiet Sun. Clearly, further study is required with both new simulations and observations to shed some light into these issues.

The authors are grateful to B.W. Lites for providing us with the ASP observations. 


\section{REFERENCES}

Bellot Rubio, L. R., Rodríguez Hidalgo, I., Collados, M., Khomenko, E., \& Ruiz Cobo, B. 2001, ApJ, 560, 1010

Carlsson, M., Stein, R. F., Nordlund, A., \& Scharmer, G. B. 2004, ApJ, 610, L137

Domínguez Cerdeña, I., Kneer, F., \& Sánchez Almeida, J. 2003, ApJ, 582, L55

Elmore, D. F., Lites, B. W., Tomczyk, S., Skumanich, A., Dunn, R. B., , Schuenke, J. A., Streander, K. V., Leach, T. W., Chambellan, C. W., Hull, \& Lacey, L. B. 1992, in Proc SPIE, Vol. 1746, 22

Emonet, T., \& Cattaneo, F. 2001, ApJ, 560, L197

Khomenko, E. V., Collados, M., Solanki, S. K., Lagg, A., \& Trujillo Bueno, J. 2003, A\&A, 408, 1115

Lites, B. W. 1996, Solar Phys., 163, 223

Lites, B. W., \& Socas-Navarro, H. 2004, ApJ, 613, 600

Ruiz Cobo, B., \& del Toro Iniesta, J. C. 1992, ApJ, 398, 375

Sánchez Almeida, J., \& Lites, B. W. 2000, ApJ, 532,1215

Sankarasubramanian, K., Elmore, D. F., Lites, B. W., Sigwarth, M., Rimmele, T. R., Hegwer, S. L., Gregory, S., Streander, K. V., Wilkins, L. M., Richards, K., \& Berst, C. 2003, in Polarimetry in Astronomy. Edited by Silvano Fineschi . Proceedings of the SPIE, Volume 4843, pp. 414-424 (2003)., 414-424

Socas-Navarro, H. 2001, in ASP Conf. Ser. 236: Advanced Solar Polarimetry - Theory, Observation, and Instrumentation, 487

Socas-Navarro, H., \& Sánchez Almeida, J. 2002, ApJ, 565, 1323

Socas-Navarro, H., \& Sánchez Almeida, J. 2003, ApJ, 593, 581

This 2-column preprint was prepared with the AAS LATEX macros v5.2. 

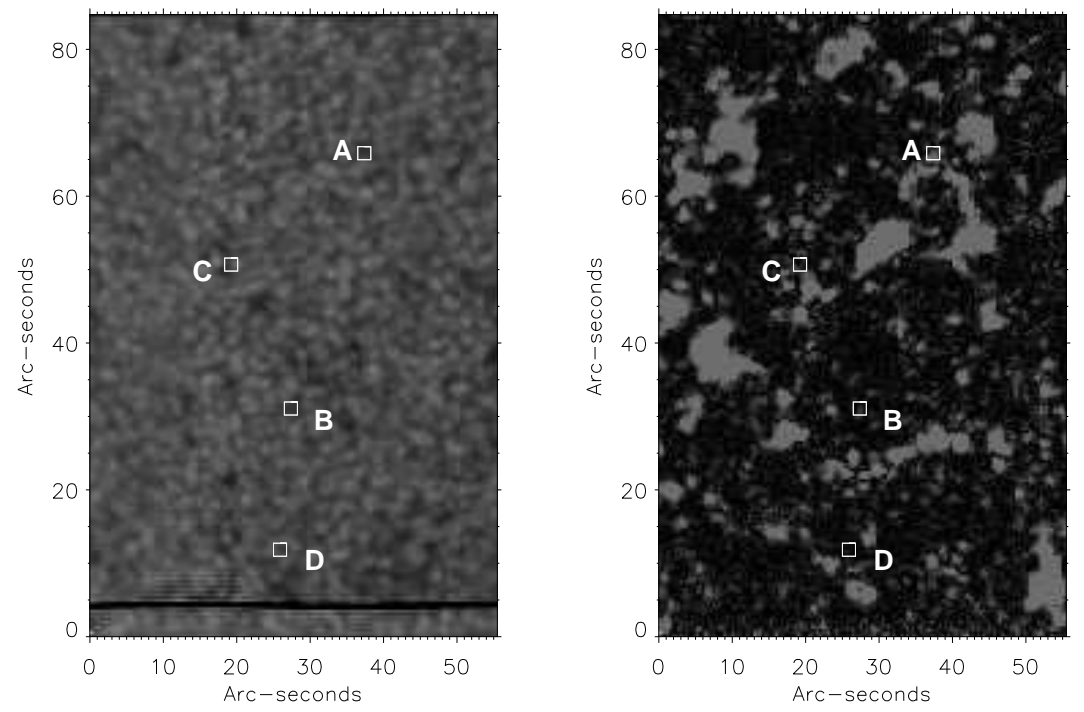

Fig. 1.- Maps of the observed region showing the location of the supersonic upflows. Left: Photospheric continuum. Right: Degree of circular polarization (V/I integrated over a $130 \mathrm{~m} \AA$ bandwidth) saturated at 0.003. White squares mark the position of interesting profiles (see text). 

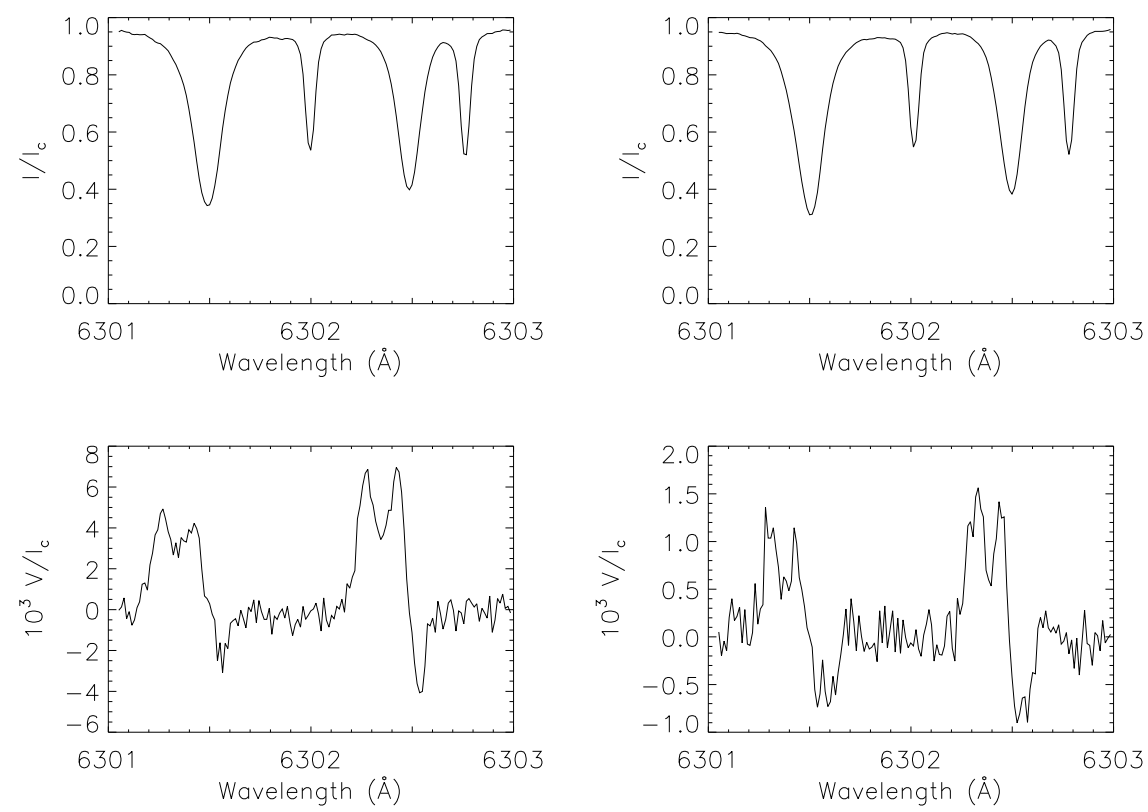

Fig. 2.- Individual Stokes $I$ (upper panels) and $V$ (lower panels) spectra of the Fe I lines at 6301.5 and $6302.5 \AA$ A. The narrow lines at 6302.0 and 6302.8 are telluric. The profiles correspond to regions A (left panels) and B (right panels), and have been normalized to the local continuum intensity $I_{c}$.
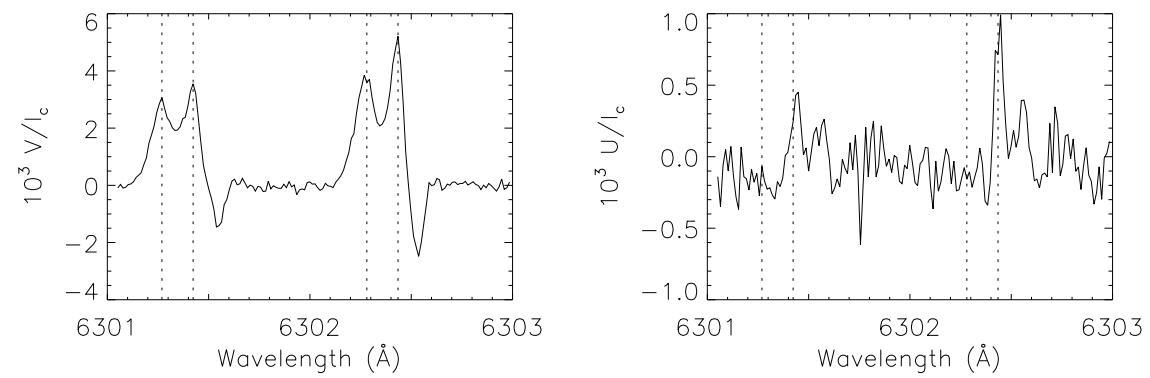

Fig. 3.- Spatial average of Stokes $V$ (left) and $U$ (right) profiles in region A. The average has been computed over a $4 \times 4$ pixel box. The vertical lines mark the positions of the Stokes $V$ blue peaks. 

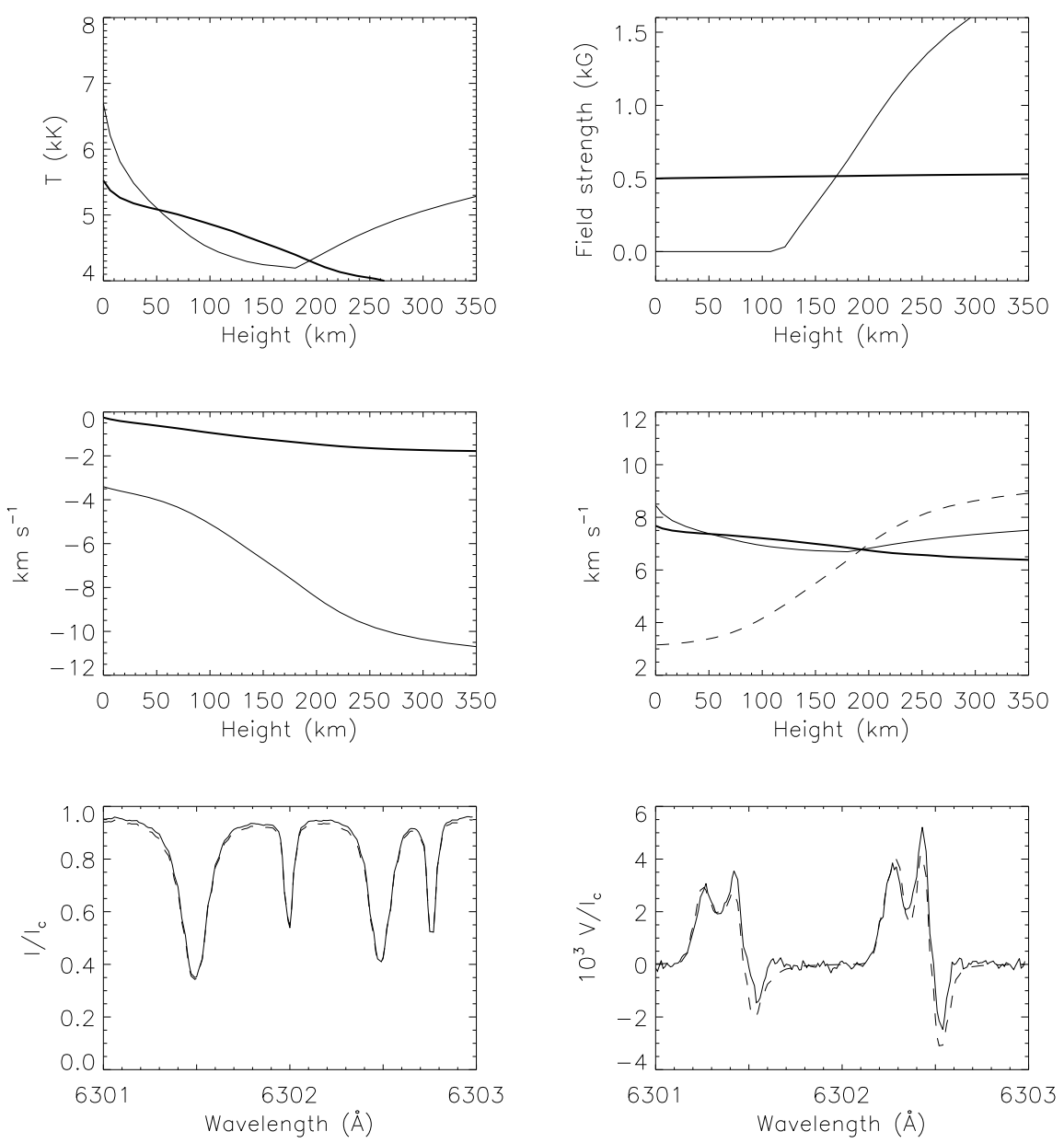

Fig. 4.- Model atmospheres (top and middle panels) and fits (bottom panels) obtained from a 2-component inversion. The thick (thin) line represents physical variables in the normal (upflowing) component. The middle panels show the line of sight velocities (left) and the sound speed (right) in each atmospheric component. The dashed line in the middle right panel is the relative velocity between the two components. The bottom panels show the observed Stokes $I$ and $V$ profiles (solid) and the fits provided by the code (dashed). The magnetic filling factor retrieved is $\alpha=0.95$. The relative occupation of the normal and upflowing components are 0.7 and 0.3 , respectively. 